\title{
EL APRENDIZAJE ORGANIZACIONAL (AO) Y EL DESEMPEÑO EMPRESARIAL BAJO EL ENFOQUE DE LAS CAPACIDADES DINÁMICAS DE APRENDIZAJE
}

\author{
Organizational Learning $(O L)$ and business performance adopting the approach of dynamic \\ learning capabilities
}

Julio Albeiro Londoño-Patiño

MSc en Gestión de la Innovación Tecnológica, Cooperación y Desarrollo Regional. Servicio Nacional de Aprendizaje-SENA, Medellín-Colombia, juliolondono1233@gmail.com

\author{
Carlos Alberto Acevedo-Álvarez \\ MSc en Gestión Tecnológica. Instituto tecnológico Metropolitano, Medellín-Colombia, \\ carlosacevedo@itm.edu.co
}

\begin{abstract}
Cómo referenciar / How to cite
Londoño-Patiño, J. y Acevedo-Álvarez, C. (2018). El aprendizaje organizacional (AO) y el desempeño empresarial bajo el enfoque de las capacidades dinámicas de aprendizaje, Revista CEA, 4(7), 103-118. https://doi.org/10.22430/24223182.762
\end{abstract}

Recibido: 30 de junio de 2017

Aceptado: 30 de septiembre de 2017

\section{Resumen}

El objetivo de este trabajo es presentar una reflexión sobre la relación entre el aprendizaje organizacional y el desempeño organizacional, bajo el enfoque de las capacidades dinámicas de aprendizaje, considerando las diversas tipologías que han sido planteadas por diferentes estudios para clasificar el aprendizaje organizacional que ocurre en las organizaciones, según su alcance colectivo o su nivel individual. Con base en estas tipologías, se consideran los factores que condicionan el desarrollo del aprendizaje con el desempeño empresarial, permitiendo encontrar las razones por las cuales las organizaciones deben asumir el aprendizaje como una plataforma de competitividad y permanencia en el tiempo como medida de mantenibilidad en entornos dinámicos y siempre cambiantes.

En términos metodológicos, para relacionar el $\mathrm{AO}$, las capacidades dinámicas de aprendizaje y el desempeño, se realizó una revisión de la literatura de orden descriptiva por periodo de tiempo, usando como referencia conceptos relacionados a la organización que aprende, partiendo desde el tipo de aprendizaje organizacional y el entorno que lo rodea (individual y colectivo); por último, se realizan unas consideraciones del aprendizaje organizacional y el desempeño empresarial que destaca la importancia del AO bajo el enfoque de las capacidades dinámicas y su incidencia en el desempeño, donde se evidencia su importancia en la competitividad empresarial desde sus diferentes niveles y estructuras.

Palabras clave: aprendizaje organizacional, desempeño empresarial, capacidades dinámicas. 


\begin{abstract}
This work aims at presenting a reflection on the relationship between organizational learning and performance adopting the approach of dynamic learning capabilities. It considers different typologies that have been proposed in diverse studies to classify organizational learning by collective scope or individual level. Based on such typologies, the factors that affect the development of organizational learning are selected. This enabled to find the reasons why organizations should view learning as a platform for competitiveness and permanence over time. It is also a measure to ensure maintainability in dynamic and everchanging environments.
\end{abstract}

Regarding the method, a descriptive literature review of specific time periods was conducted to link OL, dynamic learning capabilities and performance. It used concepts related to the learning organization as a reference. In addition, it was based on the type of organizational learning and the (individual and collective) surrounding environment. Some considerations on organizational learning and business performance highlight the importance of OL for the approach of dynamic learning capabilities and its influence on performance. Finally, the significance of $\mathrm{OL}$ in business competitiveness was demonstrated at different levels and structures.

Keywords: Organizational learning, business performance, dynamic capabilities.

\section{INTRODUCCIÓN}

Actualmente existe controversia en torno a la relación que se establece entre el aprendizaje y el desempeño de la organización. Algunos autores se muestran reticentes para reconocer las cualidades positivas de esta relación o adoptan una postura neutral: Argyris y Schön
(1978), Huber (1991), Leonard-Barton (1992), Levitt y March (1988); otros reconocen abiertamente la existencia de una relación positiva entre estos elementos: Calantone, Cavusgil, y Zhao (2002), Fiol y Lyles (1985), Prieto (2003), Senge (1992) y Stewart (1991). Las empresas obtienen ventajas competitivas sostenibles mediante la ejecución de estrategias que impulsan sus fuerzas internas, neutralizando las amenazas exteriores y disminuyendo las debilidades internas (Barney, 1991).

La revisión conceptual de este enfoque, su relevancia y aportes con respecto al desempeño y la gestión empresarial basados en el aprendizaje organizacional es la contribución que se pretenderá materializar en este artículo, igualmente, reflexionar en las tipologías del $A O$ y en algunos aspectos que permitan aportar claridad a la significancia en el desarrollo de las capacidades dinámicas, que son el puente entre los saberes adquiridos en el aprendizaje y el desempeño final de las organizaciones en los ambientes turbulentos actuales.

\section{MARCO TEÓRICO}

\section{Capacidades Dinámicas de Aprendizaje (CDA)}

El concepto de las capacidades dinámicas se atribuye a Teece y Pisano (1994), donde se refieren, en primer término, a los cambios en el entorno de las organizaciones; y en segundo lugar, hacen hincapié en el papel clave de la gestión estratégica de las habilidades de las competencias funcionales de la organización hacia el entorno cambiante. Mertens y Palomares (2006) enmarcan que la visión dinámica es reconocer que las capacidades dinámicas son la suma de un proceso a través del cual las organizaciones pueden escalar hacia productos y servicios mejorados. Barreto (2011) define la capacidad dinámica como el 
potencial de la empresa para resolver problema sistemáticamente, detectando amenazas y oportunidades, tomando decisiones oportunas.

Entre tanto, para Garzón (2015), las capacidades dinámicas de aprendizaje son la potencialidad (inimitable) de la empresa para generar nuevos saberes organizacionales a partir de una continua creación, integración y reconstrucción de sus competencias básicas, que incluyen la habilidad de la empresa para conseguir o mantener las ventajas competitivas.

En las definiciones se identifican dos aspectos comunes en ellas: la capacidad de la empresa en adaptarse y la capacidad de aprender en entornos cambiantes; además, la importancia en las organizaciones de lograr a lo largo del tiempo el ajuste estratégico en forma dinámica entre los ambientes externos e internos, para un mejor desempeño.

\section{La organización que aprende}

Tradicionalmente, la organización que aprende se fundamenta en las experiencias particulares desarrolladas en los entornos que operan y no sustentan su aprendizaje en fuentes argumentativas que permitan respaldar la generalización de sus ideas, en diferentes tipos de comportamiento empresarial. Para Wang y Huang (2013), las revisiones documentales de aprendizaje de la organización han demostrado que existen confusiones conceptuales respecto a lo que una organización aprende. Esta confusión se afianza por el pequeño número de instrumentos disponibles para la medición de los fenómenos de $\mathrm{AO}$, que dificulta aún más el desarrollo de estudios empíricos; esta dicotomía según Tsang (1991) no debería existir, sino que ambos enfoques deberían complementarse para lograr construir teorías rigurosas desde el punto de vista científico y empresarial.

\section{Tipos de aprendizaje organizacional}

En los tipos de aprendizaje que se presentan en las organizaciones se destacan dos criterios de clasificación que han sido ampliamente estudiados: el aprendizaje individual y el aprendizaje colectivo.

\section{- Aprendizaje individual}

El aprendizaje grupal es el puente entre el aprendizaje individual y organizativo, sin embargo, aunque la utilización de los equipos de trabajo como unidades básicas en las organizaciones no constituyen un fenómeno reciente, el reconocimiento de su importancia en la creación de «capital intelectual» es más tácito que explícito (Cegarra \& Rodrigo, 2004). El aprendizaje individual se caracteriza hacia el nuevo conocimiento; las personas desarrollan la capacidad de aprender y no de llenarse de contenidos para así modificar su interpretación de las cosas, olvidar la información inútil y estar abiertos a otros saberes (Garzón \& Fischer, 2008).

La trascendencia que tiene el aprendizaje individual en el aprendizaje organizacional puede considerarse, como indica Kim (1993); la importancia del aprendizaje individual en las organizaciones es evidente porque las organizaciones están compuestas de personas y sutil porque las organizaciones aprenden de forma independiente de cada individuo, pero no independiente de todos ellos, lo que fundamenta su relevancia para el desarrollo organizacional. Sin embargo, Antonacopoulou (2006) establece que la relación entre el aprendizaje individual y organizacional es uno de los problemas no resueltos en los debates actuales de $\mathrm{AO}$.

El aprendizaje caracterizado por ser individual y adaptativo, dirigido a formar los empleados según las necesidades de la organización, es de tal manera que fortalece la estabilidad y permanecía de los procedimientos y rutinas 
estandarizadas, persigue la liberación del individuo de manera que potencie su capacidad para crear valor (Alcover \& Gil, 2002). Finalmente, los contextos de apoyo y respaldo en la política de aprendizaje reflejan la identidad de la organización y trasciende a niveles superiores, donde el análisis y la captura de estas políticas definen la identidad profesional de los individuos reflejando sus esfuerzos para encajar en el contexto laboral. Por lo tanto, la identidad de lo aprendido se manifiesta al ser un líder organizacional y sus actuaciones desprenden de hallazgos que afectan considerablemente la naturaleza percibida y el enfoque de aprendizaje en sí mismo (Antonacopoulou, 2006).

El aprendizaje será siempre personal y se fundamenta en los saberes previos que el individuo posea como repositorio de conocimientos que, mediante asociaciones con su entorno y rutinas organizacionales, amplía y profundiza proporcionando a las organizaciones nuevos saberes que correctamente dirigidos permiten la sostenibilidad y permanencia de las empresas en mercados dinámicos y competitivos.

\section{- Aprendizaje colectivo}

El contenido del aprendizaje colectivo se fundamenta en el desarrollo de estándares, los procedimientos, las rutinas de trabajo, el establecimiento de lo que es correcto o incorrecto para lograr resultados superiores, y da significado a todo aquello que la organización explore o hace (Gore \& Vázquez, 2003). Sin embargo, diversos estudios (Argyris, 1977a \& Argyris, 1977b; Schumpeter, 1949 y Hedberg, 1981) se centran en los beneficios del trabajo en grupo. Por lo tanto, es recomendable lograr una atmósfera que propicie que los integrantes de los equipos de trabajo o grupos abran su mente hacia lo que ha de desarrollarse, para que obtengan de esta manera las bases para que el mensaje que se desea transmitir sea comprendido, retenido y aprehendido.

Sobre la base de estas consideraciones se puede afirmar que el contexto colectivo del aprendizaje afianza el capital intelectual de la empresa e influyen positivamente sobre el desempeño en ambientes competitivos (Cegarra \& Rodrigo, 2004).

Garzón y Fischer (2008), afirman que la experiencia empresarial demuestra que el nivel superior intelectual de los integrantes del aprendizaje colectivo desarrollado en trabajos colaborativos, es potencialmente superior al del aprendizaje individual. Según Pei (2008), las organizaciones que apoyan en forma favorable la cultura de aprendizaje organizacional dedican una atención especial en promover el intercambio de conocimientos. Los individuos con mayor capacidad de aprendizaje (aquellos de alto nivel de formación académica) están más animados a contribuir a la base de conocimiento de la organización bajo la cultura que se propició para el intercambio de conocimientos y dando énfasis al reconocimiento (Pei, 2008).

Mientras exista interés por conocimiento en la organización, el aprendizaje colectivo será una forma de convivencia y este se caracterizará por ser un proceso de transacción, donde se ve un intercambio de documentos $\mathrm{o}$ de conocimiento explícito, mientras que si existe interés en la creación de conocimiento, los grupos colaborativos crean el conocimiento social mediante la convivencia de los conceptos aprendidos (Cortina \& Santisteban, 2011).

Villa y Yaniz (1999) establecen que en el momento que el aprendizaje colectivo se apropia del concomimiento, se generan resultados significativos, además que sus integrantes se profesionalizan con mayor rapidez. Para Senge (1992) el aprendizaje colectivo o en equipo es el proceso que una vez 
alineado a la estrategia es capaz de desarrollar la capacidad de crear resultados que realmente se desean.

Cortina y Santisteban ( 2011) confirman que una organización que basa su trabajo en aprendizaje colectivo, es la que va a desarrollar las potencialidades de los individuos, no individualmente sino socialmente. Es muy satisfactorio trabajar en un equipo en el que todos se tienen confianza y se sienten unidos por un sentido de propósito común.

Finalmente, el aprendizaje colectivo no existe sin el aprendizaje individual y solo depende de la apropiación de los conocimientos por parte de las personas que integran el colectivo, el equipo de trabajo o grupo de estudio que generarán competencias distintivas y significativos que perduren y cambien la orientación de la empresa; y se convierte en un aspecto fundamental la necesidad de crear condiciones y mecanismos para la construcción de estos colectivos, equipos o grupos orientados al aprendizaje (Garzón \& Fischer, 2008).

\section{Aprendizaje según la organización}

Ausubel (1999) plantea que el aprendizaje depende de la estructura cognitiva previa que se relaciona con la nueva información, debe entenderse por "estructura cognitiva» al conjunto de conceptos e ideas que un individuo posee en un determinado campo del conocimiento, así como su organización. Es decir que un tipo de aprendizaje según la organización se fundamenta en el concepto del aprendizaje mismo, la forma como se aprende y todo lo relacionado con la gestión del conocimiento de ese aprendizaje. Así, desde los trabajos iniciales de Cyert y March (1963); Cangelosi y Dill (1965); Argyris y Schön (1978); Crossan, Lane, y White, (1999); (Zietsma, Branzei, y Vertinsky (2002) y Castañeda y Pérez (2005), han sido múltiples las investigaciones de este proceso en las organizaciones: aprendizaje, generación de conocimiento, adquisición, asimilación y aplicación de conocimiento en las organizaciones pero aún no entregan resultados relevantes y han terminado en escuelas académicas que generan confusión en las organizaciones. Estas escuelas han propuesto diferentes enfoques teóricos que plantean una serie de etapas o procesos, a través de los cuales se manifiesta el aprendizaje según la organización, pero, aunque difieren en la forma de agrupar sus enfoques, hay algunos puntos de coincidencia; a continuación, se relacionan en la Tabla 1.

Por otro lado, en el campo de las capacidades dinámicas de $\mathrm{AO}$, la literatura muestra un grado de ambigüedad entre los conceptos más generales de los recursos, las competencias y las capacidades dinámicas generando un uso inexacto de la terminología utilizada para describir determinados fenómenos. Parte de la confusión en torno a las capacidades dinámicas resulta directamente de las abundantes definiciones utilizadas por los diferentes autores (Garzón, 2015). En lo único que se coincide y es característico del aprendizaje organizacional es el proceso mediante el cual las entidades, a partir de individuos, adquieren, construyen y transfieren conocimiento independiente del tipo de organización.

De acuerdo con lo anterior, los tipos de aprendizaje según la organización están direccionados por la capacidad de aprendizaje $y$ se valora como una variable multidimensional, entre ellas, el nivel de aprendizaje y la cultura constituyen las dimensiones representativas (Garzón, 2005). Esta multidimensionalidad se realiza mediante las diferentes actividades que interactúan en la cotidianidad organizacional y se puede considerar que son inherentes a cualquier tipo de organización. 
Tabla 1. Enfoques del aprendizaje según la organización

Table 1. Approaches to learning according to the organization

\begin{tabular}{|c|c|c|c|c|c|}
\hline Autores & \multicolumn{5}{|c|}{ Enfoques del aprendizaje según la organización } \\
\hline Modelo propuesto & $\begin{array}{c}\text { Adquisición de } \\
\text { conocimiento } \\
\text { (interna y externa) }\end{array}$ & \multicolumn{2}{|c|}{$\begin{array}{l}\text { Asimilación de conocimiento } \\
\text { (interpretación y distribución) }\end{array}$} & \multicolumn{2}{|c|}{$\begin{array}{l}\text { Memoria organizativa } \\
\text { (almacenamiento y } \\
\text { recuperación) }\end{array}$} \\
\hline Daft y Weick (1984) & $\begin{array}{c}\text { Escaneo (recolectar } \\
\text { datos) }\end{array}$ & \multicolumn{2}{|c|}{ Interpretación (dar significado) } & \multicolumn{2}{|c|}{ Aprendizaje (actuar) } \\
\hline Huber (1991) & $\begin{array}{l}\text { Adquisición de } \\
\text { conocimiento }\end{array}$ & $\begin{array}{l}\text { Distribución e } \\
\text { interpretación } \\
\text { de información }\end{array}$ & $\begin{array}{l}\text { Interpretación } \\
\text { de información }\end{array}$ & \multicolumn{2}{|c|}{ Memoria organizativa } \\
\hline Dixon (1992) & $\begin{array}{l}\text { Adquisición de } \\
\text { conocimiento }\end{array}$ & $\begin{array}{l}\text { Diseminación } \\
\text { de información }\end{array}$ & $\begin{array}{l}\text { Creación de } \\
\text { significado }\end{array}$ & $\begin{array}{c}\text { Memoria } \\
\text { organizativa }\end{array}$ & $\begin{array}{l}\text { Recuperación } \\
\text { de información }\end{array}$ \\
\hline $\begin{array}{l}\text { Sinkula (1994); Slater y } \\
\text { Narver (1995) }\end{array}$ & $\begin{array}{l}\text { Adquisición de } \\
\text { información }\end{array}$ & $\begin{array}{l}\text { Distribución de } \\
\text { información }\end{array}$ & $\begin{array}{c}\text { Interpretación } \\
\text { compartida }\end{array}$ & & \\
\hline Nevis et al. (1995) & $\begin{array}{c}\text { Adquirir } \\
\text { conocimiento }\end{array}$ & \multicolumn{2}{|c|}{ Compartir conocimiento } & \multicolumn{2}{|c|}{ Utilizar conocimiento } \\
\hline Crossan et al. (1999) & Intuición & Interpretación & Integración & \multicolumn{2}{|c|}{ Institucionalización } \\
\hline Jerez (2001) & Adquisición & Interpretación & Integración & \multicolumn{2}{|c|}{ Memoria organizativa } \\
\hline Schulz (2001) & $\begin{array}{c}\text { Recolección de } \\
\text { nuevo conocimiento } \\
\text { (exploración) }\end{array}$ & \multicolumn{2}{|c|}{ Codificación de conocimiento } & \multicolumn{2}{|c|}{$\begin{array}{c}\text { Combinación de } \\
\text { conocimiento antiguo } \\
\text { (explotación) }\end{array}$} \\
\hline Tippins y Sohi (2003) & $\begin{array}{l}\text { Adquisición de } \\
\text { información }\end{array}$ & $\begin{array}{l}\text { Diseminación } \\
\text { de información }\end{array}$ & $\begin{array}{c}\text { Interpretación } \\
\text { compartida } \\
\end{array}$ & $\begin{array}{c}\text { Memoria } \\
\text { declarativa }\end{array}$ & $\begin{array}{c}\text { Memoria } \\
\text { procedimental }\end{array}$ \\
\hline $\begin{array}{l}\text { Argote y Miron-Spektor } \\
(2011)\end{array}$ & $\begin{array}{c}\text { Creación de } \\
\text { conocimiento }\end{array}$ & \multicolumn{2}{|c|}{ Transferencia de conocimiento } & \multicolumn{2}{|c|}{ Retención de conocimiento } \\
\hline
\end{tabular}

Así mismo, el enfoque de los recursos y las capacidades señala que el valor de la organización y su posición competitiva está marcada por la cantidad y características de los recursos y capacidades que la organización transforma en aprendizaje y las pueda utilizar (Garzón, 2015). El aprendizaje, según la organización, establece procesos que facilitan el desarrollo de las destrezas basadas en aptitudes y características personales, como la responsabilidad, la creatividad, iniciativa, capacidad de discusión, análisis y solución de problemas (Schein, 2004).

En definitiva, los tipos de aprendizaje según la organización aún es un tema de estudio e investigación, pero lo cierto es que la interacción dinámica entre las fuentes que lo proveen, determinan la capacidad de aprendizaje de las organizaciones, cuyos efectos sobre los resultados de la organización son mediados por la gestión del conocimiento y que con una correcta dirección, esos resultados sean superiores a lo esperado (Garzón, 2005).

\section{Aprendizaje según la interorganización}

El aprendizaje organizacional es un campo de investigación académica y de práctica profesional con un desarrollo relativamente reciente (Garzón \& Fischer, 2008). El constante cambio y evolución de los entornos económicos exige a las organizaciones un desarrollo competitivo, que les permita desarrollar tanto las capacidades como los 
recursos necesarios para producir, lográndose esto a través de las capacidades dinámicas de aprendizaje que son las que permiten la creación de conocimiento.

Bajo este concepto, el aprendizaje interorganizativo se basa en las relaciones y colaboraciones que tienen diferentes organizaciones y con las que se crea un nuevo conocimiento necesario para estas. La obtención de dicho conocimiento se dificulta por el acelerado ritmo en el que suceden los cambios, pero con el aprendizaje interorganizativo se facilita la creación de conocimiento a través del establecimiento de acuerdos de cooperación y apoyo entre organizaciones, en los que aprenden unas de otras, creando alianzas estratégicas que permiten acceder a los recursos y a las capacidades complementarias que tienen otras empresas.

Sin embargo, aún se presenta una brecha en los términos que la definen, sobre cómo este se crea, transfiere y aplica el conocimiento en el nivel interorganizacional, factor que se convierten en pilar fundamental en la sostenibilidad de las organizaciones en mercados dinámicos (Garzón \& Fischer, 2008). Entornos donde existe un creciente interés en la naturaleza de vínculos interorganizacionales y sus implicaciones para crear y compartir conocimientos (Ahuja, 2000; Hajidimitriou, Sklavounos \& Rotsios, (2000); Howells, 2006; Powell, White, Owen-smith \& Koput, (2005); Uzzi, 1999; Zaheer \& McEvily, 1999).

Basados en los conceptos desarrollados, se puede establecer la diferencia de los lazos interorganizacionales en una serie de dimensiones donde el carácter de las relaciones sociales entre los actores, incluyendo orientaciones de esos actores a la relación de intercambio y la regulación de la relación; y su temporalidad dimensional, es decir, su frecuencia de uso y la duración de la relación (Mariotti \& Delbridge, 2012). Estas dimensiones están interrelacionadas entre sí. Se inicia con la dimensión de intervención, considerada la de mayor importancia, pues en ella se realiza el diagnóstico a través de las variables que influyen en el aprendizaje de la organización.

A partir de los resultados de la intervención, se continúa con la dimensión de generación de relaciones, encaminada a determinar qué interrelaciones deberán ocurrir entre las personas de la organización que la integran en función de la información y conocimiento que poseen, para promover el intercambio e incrementar la capacidad de aprendizaje para la organización. La siguiente dimensión formaliza el proceso de aprendizaje a partir de las situaciones y acciones definidas. Se utiliza el término formalización para dar sentido de formación al aprendizaje, incluye todo tipo de acción de formación, no solo la capacitación tradicional mediante cursos sino cualquier acción en la cual los individuos se relacionen e interactúen, y de esta forma, compartan información útil o construyan conocimientos en conjunto y logren desarrollar un aprendizaje colaborativo. En la última dimensión se presenta la incidencia, esta permite conocer cómo el aprendizaje ha incidido en la mejora del desempeño de la organización, se revalúa la capacidad de aprendizaje para comprobar en qué medida lo han incorporado a la organización y se analiza el comportamiento de los indicadores (Rodríguez, 2016).

Como consecuencia, en la nueva economía el conocimiento organizativo adquiere un papel singular como fuente de ventaja competitiva sostenida para la organización. Por ello, las empresas deben saber cómo gestionar de modo eficaz y eficiente este recurso estratégico y cómo renovar su fuente de ventaja competitiva a través del aprendizaje organizativo e interorganizativo. Finalmente, en un contexto internacional, el análisis del 
aprendizaje organizativo se torna más complejo aun cuando se añade un nivel adicional: el interorganizativo (Ordóñez, 2003).

Las capacidades dinámicas de aprendizaje en la interorganización muestran características comunes para varias organizaciones, lo cual implica que son equifinales, es decir, esas capacidades tendrán una determinada creación y evolución a través de distintas trayectorias, pero llegarán a un estado en el que presentan los mismos atributos claves (Garzón, 2015). En este orden de ideas, se requiere determinar la existencia de atributos claves comunes, lo cual implica que esas rutinas son fácilmente sustituibles por otras. Se destacan los conceptos de aprendizaje, la cognición y los mecanismos de aprendizaje que presentan Eisenhardt y Martin (2000), quienes muestran cómo estudios sobre prácticas repetidas en adquisición de empresas (en este caso la adquisición es una capacidad dinámica) generarán una acumulación de conocimiento que logra desempeños exitosos para la organización.

Sin duda, en un contexto internacional, como es el caso de las multinacionales, el proceso de aprendizaje se vuelve aún más complejo. Por un lado, la matriz o las filiales puede que no dispongan de la capacidad necesaria para transmitir conocimiento entre unidades. Por otro, una centralización en la matriz podría dificultar la capacidad de reacción de las filiales ante las demandas de sus entornos locales (Lyles \& Salk, 1996). Por último, como lo expresan Cohen \& Levinthal (1990), las filiales podrían carecer de la capacidad de absorción necesaria para aprender de los entornos, es decir, podrían no disponer de la habilidad organizativa requerida para reconocer el valor del conocimiento externo nuevo, asimilarlo y aplicado con fines comerciales en sus relaciones interorganizativas (Ordóñez, 2003).

\section{Aprendizaje según interorganización y las Capacidades Dinámicas Relacionales (DRC)}

Desde una perspectiva relacional, las capacidades dinámicas relacionales son el resultado de diferentes dimensiones (técnicas, tecnológicas, humanas, entre otras), que permiten materializar objetivos conjuntos e interfuncionales; donde la capacidad relacional humana refuerza la gestión de las relaciones de cooperación, siendo un precursor del aprendizaje interactivo. Las DRC entran en juego en el desempeño de las organizaciones, con el establecimiento de estructuras y estrategias distintivas que fomentan la creación y apropiación del conocimiento (Weissenberger \& Schwenk, 2009).

En el contexto organizacional, Lorenzoni y Lipparini (1999) demostraron cómo las capacidades relacionales implican el establecimiento de una interacción fluida, con el objetivo de establecer y mejorar la base de recursos de una empresa. El establecimiento de relaciones horizontales y verticales ha dado claridad sobre el impacto positivo de las capacidades relacionales en la adquisición de nuevos conocimientos, y reafirma la importancia de estas en el rendimiento o desempeño empresarial (Zollo \& Winter, 2002; Weissenberger \& Schwenk, 2009; Fitjar \& Rodríguez-Pose, 2013).

Bajo estos conceptos, los procesos de aprendizaje entre organizaciones que activan las capacidades dinámicas relacionales contribuyen al logro de mejores desempeños, siempre en conjunción con objetivos comunes y estrategias distintivas que permitan destrezas y conocimientos especializados para lograr mayores beneficios. Esto requiere canales abiertos continuos y relaciones fluidas (Salman \& Saives, 2005). 
3. EL APRENDIZAJE ORGANIZACIONAL Y EL DESEMPEÑO EMPRESARIAL

\section{Consideraciones del aprendizaje organizacional y el desempeño empresarial}

Indiscutiblemente es de interés el análisis del impacto que puede generar la capacidad de aprendizaje sobre los resultados en las organizaciones. La definición del concepto de aprendizaje organizativo en algunos autores, sin importar su enfoque, implica que el aprendizaje se evidencia en el mejoramiento del desempeño empresarial en los mercados, ya que ocurre cuando la organización modifica su comportamiento para adaptarse al entorno, aprovechando su capacidad de absorción (Cyert \& March, 1963).

En este sentido, hay investigaciones que identifican las curvas de experiencia en la organización como la evidencia de la relación existente entre aprendizaje y desempeño, ya que demuestran como la adquisición, asimilación, repetición, acumulación, reconstrucción, reconfiguración de experiencia en la realización de una actividad que genera en el tiempo una reducción de los costos de dicha actividad (Yelle, 1979; Shrivastava, 1983;
Álvarez, 2000; Askvik, 1999; Gil \& Carrillo, 2014; Hajidimitriou et al., 2000; Heng, 2010; Lyles \& Salk, 1996). Lo que comúnmente comparte la literatura sobre la gestión es el énfasis y la necesidad de conceptos de cambio organizacional que son más adecuados para un entorno empresarial, cada vez más turbulento y complejo (Pawlowsky, 2000). El marco interpretativo para esta situación varía, sin embargo, y abarca una serie de escenarios tales como el post industrial en la sociedad, una economía de servicios y una sociedad del conocimiento. Todos estos escenarios son de captura y algunos aspectos de un cambio radical en las condiciones como miran las organizaciones y empresas los nuevos aspectos que se están produciendo (Pawlowsky, 2000).

Con la instrucción y la repetición, los trabajadores aprenden a realizar trabajos de manera más eficiente y con ello reducir el número de horas de mano de obra directa por unidad. Al igual que los trabajadores, las organizaciones aprenden. El AO implica adquirir experiencia con productos y procesos, logrando una mayor la eficiencia mediante las mejoras en los métodos administrativos y el capital intelectual (Ver Figura 1).

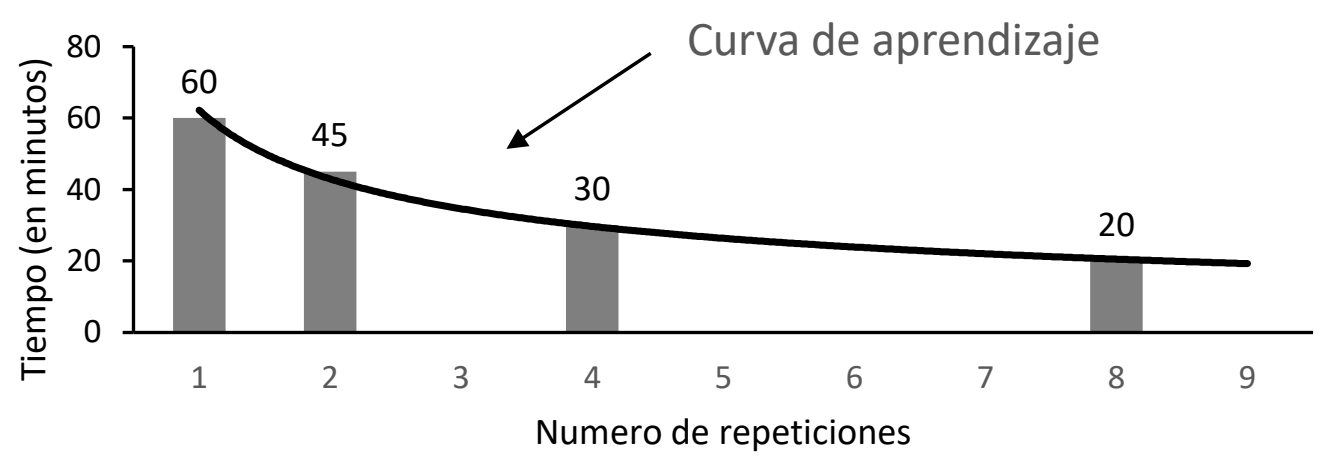

Figura1. Curva de aprendizaje organizacional

Figure 1. Organizational learning curve

Fuente: elaboración propia a partir de Yelle (1979). 
Cuando se evalúa en qué medida el rendimiento y el aprendizaje organizacional impactan la organización y la empresa, se basa en la comprensión de construcciones, tales como eficacia de la organización, cambio organizacional, el AO y la cultura organizacional (Johnson \& Bailey, 2010). Pero la dinámica del sistema considera los procesos de construcción de modelos, que se supone que debe generar un considerable aprendizaje acerca de un problema de política organizacional. No solo a nivel individual sino también a nivel colectivo. Desde el punto de vista de la consecuencia de un proceso de construcción de qué modelo de aprendizaje y desempeño podría ser establecido (Vennix \& Scheper, 1990).

Es importante señalar que, si bien la difusión de las nuevas organizaciones flexibles y de sus características más generales se realiza a escala mundial, su aplicación concreta en cada país o región precisa de procesos de adaptación e innovación organizacional en la medida que el crecimiento del ámbito informal en este tipo de organizaciones hace que su estructura sea mucho más permeable a los contextos socioculturales qué la rodean (Álvarez, 2000). Sin embargo, el aprendizaje desde un enfoque cognitivo se considera un proceso en donde el desempeño empresarial es independiente del cambio en el procedimiento empresarial, ya que es posible que una organización modifique su comportamiento aplicando adaptación al entorno sin que se presente un cambio en su estructura cognitiva (Fiol \& Lyles, 1985). En todo caso, aunque el aprendizaje no relaciona los comportamientos finales, es evidente que cuando una organización aprende, la brecha en sus comportamientos potenciales cambia (Huber, 1991), ello incrementa en forma positiva la probabilidad que la empresa se adapte en forma eficientemente las circunstancias del entorno, mejorando a largo plazo su desempeño.
Por lo tanto, las organizaciones exitosas son aquellas que articulan la estrategia y las necesidades de los empleados en todos los niveles, teniendo en cuenta lo que requieren conocer, compartir y aprender, para ejecutar dichas estrategias (Almanza, Calderón, Vargas, Casas, \& Palomares, 2016). Dicha articulación guía el despliegue de los recursos organizacionales y tecnológicos, y de las capacidades para aprovechar al máximo el conocimiento, lo cual incrementa las posibilidades de generar valor (Zack, 2007). La formación de estrategias es un espacio complejo (Mintzberg, Ahlstrand, \& Lampel, 2013). Es un diseño crítico, intuitivo, con visión y aprendizaje emergente; se trata de la transformación del aprendizaje organizacional para el mejor desempeño; debe involucrar cognición individual y social, interacción y cooperación; tiene que incluir el analizar antes y después de la organización, así como todo esto debe ser en respuesta a lo que puede ser un entorno exigente (Mintzberg, Ahlstrand, \& Lampel, 2009).

Es así como la teoría de las capacidades dinámicas de aprendizaje tiene un rol estratégico en el desempeño final de la organización con el fin de que esta pueda construir y sostener ventajas competitivas superiores a otras organizaciones.

\section{Nivel de aprendizaje y desempeño}

El dinamismo del entorno, los permanentes cambios y la consecuente incertidumbre del mercado han provocado que las organizaciones se cuestionen sobre el desarrolle estratégico convencional para entender las diferencias en el desempeño de las empresas (Cardona \& Calderón, 2006).

García, Jiménez, y Llorens (2011) expresan que dependiendo de la naturaleza del proceso existen dos niveles principales de $A O$, aunque sus nombres difieren según la literatura 
investigada; inicialmente el aprendizaje adaptativo o bucle simple permite que los errores existentes sean detectados y corregidos mediante el cambio de las rutinas de comportamiento para que, de ese modo, los errores no afloren de nuevo. Así, el desempeño organizativo se mantiene dentro de los límites establecidos por las normas y los valores existentes. La segunda, es el aprendizaje generativo, que fomenta una retroalimentación de doble bucle: por un lado, al igual que el bucle simple, permite que los errores existentes sean detectados y corregidos (Argyris \& Schön, 1978); por otro lado, y aquí radica la diferencia, conecta esos errores con los valores y las normas y, por lo tanto, las estrategias y las acciones cambian (García et al., 2011).

Finalmente, el nivel de aprendizaje se debe encaminar al mejoramiento del desempeño, así, el aprendizaje logra que las normas, percepciones y principios, se modifiquen (Argyris \& Schön, 1978). Se produce un proceso de desaprendizaje de lo aprendido para dar espacio al desarrollo de nuevas estructuras y esquemas mediante un sistema de valores alternativos, rutinas, tecnologías, objetivos y propuestas, que apoyen el AO (Fiol \& Lyles, 1985).

«Las empresas sin tecnología, pueden adquirirla; sin dinero, pueden financiarse; pero sin líderes y personal capacitado, comprometido y leal con los valores de la organización, morirán inexorablemente» (Siliceo, Casarez \& González, 1999, p. 211).

\section{DISCUSIÓN E IMPLICACIONES}

Desde los planteamientos realizados inicialmente por Teece y Pisano (1994), hasta llegar a Garzón (2015), se evidencia la importancia de las capacidades dinámicas, tanto relacionales como de aprendizaje; sin embargo, las organizaciones aún no han comprendido a plenitud su importancia, situación que se puede fundamentar por la incertidumbre que las organizaciones están viviendo por los cambios frecuentes, que a su vez genera la necesidad de compartir conocimientos para lograr una mayor competitividad y visibilidad en el ambiente organizacional. En este sentido, es importante resaltar que estos entornos conducen a las organizaciones a asociarse e interactuar en escenarios complejos que permiten una gestión de conocimientos eficiente y de alto impacto, lo cual confirma que la organización que aprende se convierte en un agente mediador para resultados empresariales mejorados.

Sin embargo, a partir de los hallazgos se puede evidenciar que no todas las organizaciones están preparadas para comportarse de esta manera; igualmente han empezado a participar en procesos de aprendizaje entre ellas, propiciando el logro de objetivos estratégicos y la superación de obstáculos, percibiendo nueva oportunidad de crecimiento. Lo anterior refuerza el interés del presente trabajo, en contribuir con una reflexión descriptiva de cómo las organizaciones crean, comparten y aplican conocimientos desde el enfoque de las capacidades dinámicas de aprendizaje.

En este orden de ideas, se pudo observar que las organizaciones aprenden y generan conocimiento, primero a partir del desarrollo de las CDA; y en segundo lugar, la forma como las organizaciones activan el proceso de aprendizaje entre ellas (DRC) (Knight \& Littleton, 2018), terminan convirtiéndose en organizaciones exitosas en las que puedan evidenciar resultados de alto impacto.

Ahora bien, no siempre el aprendizaje mejora la eficacia de la persona que aprende Huber (1991), pero sí es factor clave como organización, ya que esta no solo se compone 
de una, lo cual permite mejorar su desempeño dentro del mercado donde compite (Almanza et al., 2016).

\section{CONCLUSIONES}

La organización con baja capacidad de aprendizaje se caracteriza por su bajo desempeño, tanto en la apropiación del conocimiento adquirido, así como en la práctica.

Basada en los procesos de aprendizaje y sus diferentes formas de adquirirlo, la organización se convierte en una organización con problemas estratégicos cuando no logra ser competitiva aprovechando los conocimientos sobre las dinámicas de la empresa; caso contrario a la organización orientada al desarrollo de las capacidades dinámicas de aprendizaje, que es una organización abierta al cambio, busca nuevas oportunidades y cuenta con buenas capacidades en otros ámbitos.

En términos generales, la literatura sobre cómo aprende la organización está creciendo y permite abordar el aprendizaje como un proceso más amplio, ya que no solo considera la necesidad que tienen las empresas de ser exitosas a través de la apertura hacia nuevos conocimientos, sino también hacia el uso eficiente y refinamiento de los conocimientos que la empresa ya posee. Esto necesariamente se refleja en los resultados con la medición del desempeño.

El desempeño empresarial está directamente relacionado con el aprendizaje organizacional y este a su vez con el desarrollo de las capacidades dinámicas de aprendizaje que toda organización dentro de sus estrategias debe aplicar y fortalecer, de esta forma se puede ubicar la influencia motivacional de la cultura organizacional en el desempeño de quienes integran la organización, estos a su vez son los ejecutores claves de los cambios que generan la competitividad y sostenibilidad deseados; la apropiación del aprendizaje es la principal responsable para lograr las metas sobre la base de nuevos saberes adquiridos. Por tanto, se deduce la importancia que tiene el desarrollo de las capacidades dinámicas de aprendizaje, y en especial lo vinculado con el desempeño empresarial.

En definitiva, la interacción dinámica entre las fuentes, los niveles de aprendizaje, los saberes adquiridos y las condiciones para el aprendizaje, los conocimientos y los procesos de las capacidades dinámicas que los desarrollan determinan la capacidad de aprendizaje de las organizaciones, cuyos efectos moderados por la gestión del conocimiento determinan los resultados de la organización, el desempeño y competitividad en los mercados

Futuras investigaciones podrían abordar las interacciones que pueden presentarse entre el $\mathrm{AO}$ adquirido en relación con el desempeño empresarial. En el caso particular de las estrategias organizacionales.

\section{REFERENCIAS}

Ahuja, G. (2000). Collaboration networks, structural holes, and innovation: A longitudinal study. Administrative Science Quarterly, 45(3), 1-32.

Alcover, C. M., \& Gil, F. (2002). Creating knowledge jointly: organization and group learning. Revista de Psicología del Trabajo y de las Organizaciones, 18(2), 259-301.

Almanza, R., Calderón, P., Vargas, J. G., Casas, R., \& Palomares, F. (2016). Aprendizaje y desempeño organizacional bajo el enfoque de las teorías organizacionales. Revista de Economía \& Administración, 13(1), 1-12. 
Álvarez, A. B. (2000). Cambio organizacional y cambio en los paradigmas de la administración. Dimensión Empresarial, 48(9), 11-34.

Antonacopoulou, E. P. (2006). The Relationship between Individual and Organizational Learning. New Evidence from Managerial Learning Practices, 37(4), 455-473.

Argyris, C. (1976). Double-Loop Learning in Organizations. Harvard Business Review, 55(5), 115-125.

Argyris, C. (1977). Organizational Learning and Management Information Systems. Accounting, Organizations, and Society, 2(2), 113-123.

Argyris, C., \& Schön, D. A. (1978). Organizational Learning: A Theory of Action Perspective. The Journal of Applied Behavioral Science, 15(4), 545-548.

Askvik, S. (1999). La gerencia y el aprendizaje organizacional. Gestión y Política Pública, 8(2), 1-14.

Ausubel, P. D. (1999). Teoría del aprendizaje. Psicologia Educativa, 3(2), 1-10.

Barney, J. (1991). Firm Resources and Sustained Competitive. Journal of Management, 17(1), 99-120.

Barreto, I. (2011). Conferencia: Capacidades dinámicas: una revisión de la investigación pasada y agenda para el futuro. Portugal: Universidade Catolica Portuguesa. Recuperado de https://bit.ly/2HMKPVK

Calantone, R. J., Cavusgil, S. T., \& Zhao, Y. (2002). Learning orientation, firm innovation capability, and firm performance. Industrial Marketing Management, 31(6), 515-524.
Cangelosi, V. E., \& Dill, W. R. (1965). Organizational Learning: Observations Toward a Theory. Administrative Science Quarterly, 10(2), 175-203.

Cardona, J. A., \& Calderón, G. (2006). El impacto del aprendizaje en el rendimiento de las organizaciones. Manizales, Colombia: Universidad Nacional de Colombia. Recuperado de http://www.scielo.org.co/pdf/cadm/v19n 32/v19n32a02.pdf

Castañeda, D. I., \& Pérez, A. (2005). ¿Cómo se produce el aprendizaje individual en el aprendizaje organizacional? Una explicación más allá del proceso de intuir. Revista Interamericana de Psicología Ocupacional, 24(24), 1-2.

Cegarra, J. G., \& Rodrigo, B. (2004). Componentes de éxito del aprendizaje grupal en el capital intelectual. Investigaciones Europeas de Dirección y Economía de la Empresa, 10(2), 75-92.

Cohen, W., \& Levinthal, D. A. (1990). Absorptive Capacity: A New Perspective on Learning and Innovation. Administrative Science Quarterly, 35(1), 128-152.

Cortina, J., \& Santisteban, D. F. (2011). Perspectivas del aprendizaje organizacional como catalizador de escenarios competitivos. Revista Ciencias Estratégicas, 19(26), 247-266.

Crossan, M. M., Lane, H. W., \& White, R. E. (1999). An Organizational Learning Framework: From Intuition to Institution. Academy of Management Review, 24(3), 522-537.

Cyert, R. M., \& March, J. G. (1963). This Week' $s$ Citation Classic. Organization Science, 46(40), 1-25. 
Eisenhardt, K. M., \& Martin, J. A. (2000). Dynamic capabilities: What are they? Strategic Management Journal, 21(10/11), 1105-1121.

Fiol, M., \& Lyles, M. (1985). Organizational learning. The Academy of Management Review, 10(4), 803-813.

Fitjar, R. D., \& Rodríguez-Pose, A. (2013). Firm collaboration and modes of innovation in Norway. Research Policy, 42(1), 128-138.

García, V. J., Jiménez, M., \& Llorens, F. (2011). Influencia del nivel de aprendizaje en la innovación y desempeño organizativo: factores impulsores del aprendizaje. Revista Europea de Dirección y Económica de la Empresa, 20(1), 161-186.

Garzón, M. (2005). Niveles del aprendizaje organizacional. Facultad de Administración, 22, 1-77.

Garzón, M. (2015). Modelo de capacidades dinámicas. Dimensión Empresarial, 12(3), 111-131.

Garzón, M., \& Fischer, A. L. (2008). Modelo teórico de aprendizaje organizacional. Pensamiento and Gestión, 24, 195-224.

Gil, A. J., \& Carrillo, F. J. (2014). La creación de conocimiento en las organizaciones a partir del aprendizaje. Omnia Science, 9(3), 730-753.

Gore, E., \& Vázquez, M. (2003). Aprendizaje colectivo y capacitación laboral. Construcción Social del Conocimiento. Buenos Aires: American Commodity Distribution Association.

Hajidimitriou, Y. A., Sklavounos, N. S., \& Rotsios, K. P. (2000). The impact of trust on knowlwsgw transfer in International Business Systems. Scientific Bulletin -
Economic Sciences, 11(2), 39-49.

Hedberg, B. (1981). How Organizations Learn and Unlearn. In W. H. Nystrom P. C. Y Starbuck (Ed.), Handbook of Organizational Design (pp. 3-27).

Heng, T. M. (2010). Learning Curves and Productivity in Singapore Manufacturing lindustries. In Paper presented at the Second Annual Conference of the Academic Network for Development in Asia (ANDA) (pp. 8-10). Cambodia.

Howells, J. (2006). Intermediation and the role of intermediaries in innovation. Journal of Management Studies, 35(5), 715-728.

Huber, G. P. (1991). Organizational Learning : The Contributing Process and Literatures Organizational. Organization Science, 2(1), 1-43.

Johnson, C. G., \& Bailey, M. R. (2010). Validating an Organizational Action System Model from a Learning and Performing Perspective. Academy of Management Proceedings, 20(9), 1-34.

Kim, D. H. (1993). The Link Between Individual and Organizational Learning. Sloan Management Review, 35(1), 1-14.

Knight, S., \& Littleton, K. (2018). A discursive approach to the analysis of epistemic cognition. Learning, Culture and Social Interaction, 16(November 2017), 55-69.

Leonard-Barton, D. (1992). Core Capabilities and core Rigidities: A paradox in Managing new Product Development. Journal of Chemical Information and Modeling, 53, 1689-1699.

Levitt, B., \& March, J. (1988). Organizational Learning. Annual Review of Sociology, 14(1988), 319-340. 
López, E. (2014). Relaciones entre el aprendizaje organizativo, las estrategias de diversificación tecnológica y el desempeño empresarial: un estudio empírico en empresas manufactureras en España.

Lorenzoni, G., \& Lipparini, A. (1999). The leveraging of intrafirm relationships as a distinctive organizational capability: A longitudinal study. Strategic Management Journal, 20(June 1996), 317-338.

Lyles, M. A., \& Salk, J. E. (1996). Knowledge acquisition from foreign parents in international joint ventures: an empirical examination in the hungarian context. Journal of International Business Studies, 12(4), 877-903.

Mariotti, F., \& Delbridge, R. (2012). Overcoming Network Overload and Redundancy in Interorganizational Networks: The Roles of Potential and Latent Ties. Organization Science, 23(2), 511-528.

Mertens, L., \& Palomares, L. (2006). Capacidades dinámicas de aprendizaje en las organizaciones: ¿̇gestión de la ambigüedad y dilemas, base de la economía de aprendizaje? Seminario Internacional Globalización, Comocimiento Y Desarrollo. México: UNAM.

Mintzberg, H., Ahlstrand, B., and Lampel, J. (2009). Strategy safari: The complete guide through the wilds of strategic management. Financial Times Prentence Hall. New York.

Mintzberg, H., Ahlstrand, B., \& Lampel, J. (2013). Management? It's not what you think! ( $6^{\mathrm{a}}$ ed.). New York: Pearson UK.
Ordóñez de Pablos, P. (2003). Aprendizaje organizativo en un contexto internacional: implicaciones para la gestión del conocimiento. Ivestigaciones Europeas, 9(2), 205-216.

Pawlowsky, P. (2000). Management science and organizational learning. Management Science and Organizational Learning, 8(12), 1-45.

Pei, N. S. (2008). Enhancing Knowledge Creation in Organizations. Enhancing Knowledge Creation in Organizations.

Powell, W. W., White, D. R., Owen-smith, J., \& Koput, K. W. (2005). Network Dynamics and Field Evolution: The Growth of Interorganizational Collaboration in the Life Sciences. American Journal of Sociology, 110(4), 1132-1205.

Prieto, I. (2003). Una valorización de la gestión del conocimiento para el desarrollo de la capacidad de aprendizaje en las organizaciones: propuesta de un modelo integrador. Biblioteca virtual Miguel de Cervantes. Universidad de Valladolid.

Rodríguez, Y. S. (2016). Modelo y metodología de aprendizaje organizacional para el mejor desempeño de una organización de ciencia e innovación. Habana: acultad de Ingeniería Industrial, Instituto Superior Politécnico José Antonio Echeverría, Cujae.

Salman, N., \& Saives, A. L. (2005). Indirect networks: an intangible resource for biotechnology innovation. Resource and Management, 35(2), 203-215.

Schein, E. H. (2004). Organizational Culture and Leardership. (A Wiley Imprint, Ed.) (3rd ed.). San Francisco: Jossey Bass. 
Schumpeter, J. A. (1949). Change and the Entrepreneur. In N. B. Richard V. Clemence (Ed.), Economic Theory and Entrepreneurial History (pp. 63-84). Transaction Publishers.

Senge, P. (1992). The fifth discipline: The art and practice of the learning organization. (Granica, Ed.) (6th ed.). New York: Doubleday.

Shrivastava, P. (1983). A Typology of Organizational Learning Systems. Journal of Management Studies, 21(1), 7-28.

Siliceo, A., Casarez, D., \& González, J. L. (1999). Liderazgo, valores y cultura organizacional: hacia una organización competitiva. Cultura Organizacional y Liderazgo, 127-261.

Stewart, T. (1991). Intellectual capital is becoming corporate America's most valuable asset and can be its sharpest competitive weapon. Fortune, 1-9.

Teece, D., \& Pisano, G. (1994). The Dynamic Capabilities of Firms: an Introduction. Industrial and Corporate Change, 3(3), 537-556.

Tsang, E. (1991). Differential Regulation of Superoxide Dismutases in Plants Exposed to Environmental Stress. The Plant Cell Online, 3(8), 783-792.

Uzzi, B. (1999). Embeddedness in the making of financial capital: how social relations and networks benefit firms seeking financing. American Psychological Review, 64(3), 481-505.

Vennix, J. A. M., \& Scheper, W. J. (1990). Modeling as organizational learning: an empirical perspective. Journal of International Business Studies, 5(4), 1199-1210.
Villa, A., \& Yaniz, C. (1999). Organizational learning and professional development. Revista de Currículum y Formación del Profesorado, 1, 1-20.

Wang, Y., \& Huang, S. (2013). Organizational Learning and Human Resource : A Review of the Theory and Literatures. International Proceedings of Economics, 61(12), 56-59.

Weissenberger, M., \& Schwenk, J. (2009). Lifeblood knowledge: dynamic relational capabilities (DRC) and knowledge for firm innovativeness and competitive advantage. Measuring Business Excel, 13(2), 7-16.

Yelle, L. E. (1979). The Learning Curve: Historical Review and Comprehensive Survey. Decision Sciences, 10, 302-328.

Zack, M. H. (2007). The role of decision support systems in an indeterminate world. Decision Support Systems, 43(4), 16641674.

Zaheer, A., \& McEvily, B. (1999). Bridging ties: a source of firm heterogeneity in competitive capabilities. Strategic Management Journal, 20(5), 1133-1156.

Zietsma, C., W., Branzei, O., \& Vertinsky, I. (2002). The war of the woods: Facilitators and impediments of organizational learning processes. British Journal of Management, 13(Special), 61-74.

Zollo, M., \& Winter, S. (2002). Deliberate learning and the evolution of dynamic capabilities. Forthcoming in Organization Science Special Issue on "Knowledge, Knowing and Organization," 58(12), 1-47. 
